\title{
The role of substrate pre-stretch in post-wrinkling bifurcations
}

\section{Citation}

Auguste, Anesia, Lihua Jin, Zhigang Suo, and Ryan C. Hayward. 2014. "The Role of Substrate Pre-Stretch in Post-Wrinkling Bifurcations." Soft Matter 10 (34): 6520. doi:10.1039/c4sm01038h.

\section{Published Version}

doi:10.1039/C4SM01038H

\section{Permanent link}

http://nrs.harvard.edu/urn-3:HUL.InstRepos:13919154

\section{Terms of Use}

This article was downloaded from Harvard University's DASH repository, and is made available under the terms and conditions applicable to Open Access Policy Articles, as set forth at http:// nrs.harvard.edu/urn-3:HUL.InstRepos:dash.current.terms-of-use\#OAP

\section{Share Your Story}

The Harvard community has made this article openly available.

Please share how this access benefits you. Submit a story.

\section{Accessibility}




\title{
The role of substrate pre-stretch in post-wrinkling bifurcations
}

\author{
Anesia Auguste ${ }^{1,}$, Lihua Jin ${ }^{2,}$, Zhigang Suo ${ }^{2, *}$, Ryan C. Hayward ${ }^{1, *}$
}

1 Department of Polymer Science \& Engineering, University of Massachusetts, Amherst, MA, 01003, USA.*E-mail: rhayward@mail.pse.umass.edu

2 School of Engineering and Applied Sciences, Kavli Institute for Nanobio Science and Technology, Harvard University, Cambridge, MA, 02138, USA. *E-mail: suo@seas.harvard.edu

† These authors contributed equally to this work

\begin{abstract}
When a stiff film on a soft substrate is compressed, the surface of the film forms wrinkles, with tunable wavelengths and amplitudes that enable a variety of applications. As the compressive strain increases, the film undergoes post-wrinkling bifurcations, leading to period doubling and eventually to formation of localized folds or ridges. Here we study the post-wrinkling bifurcations in films on pre-stretched substrates. Through a combination of experiments and simulations, we demonstrate that pre-stretched substrates not only show substantial shifts in the critical strain for the onset of post-wrinkling bifurcations, but also exhibit qualitatively different post-wrinkled states. In particular, we report on the stabilization of wrinkles in films on pre-tensioned substrates and the emergence of 'chaotic' morphologies in films on pre-compressed substrates.
\end{abstract}

\section{Introduction}

Wrinkles form when a thin stiff film supported on a soft substrate is compressed beyond a critical strain. When the compression is slightly above the critical strain, the wrinkles are periodic. The wavelength of the wrinkles is determined by a balance 
between the deformation of the substrate, which favors short wavelengths, and the bending of the film, which favors long wavelengths ${ }^{[1,2]}$. By controlling the onset and evolution of wrinkles in a number of different material systems, researchers have demonstrated a wide range of applications. These include the fabrication of materials with changeable wetting ${ }^{[3-6]}$, adhesive $\mathrm{e}^{[7-9]}$, and optical properties ${ }^{[6,10,11]}$, the measurement of mechanical characteristics ${ }^{[12-17]}$ of thin films and soft substrates, and the use of wrinkles as templates for self-assembly ${ }^{[18-20]}$, in microfluidic ${ }^{[21-23]}$, photovoltaic ${ }^{[24-26]}$ and flexible electronic devices ${ }^{[14,27-29]}$, and for alignment of cultured cells ${ }^{[20,30,31]}$.

When compressed sufficiently far beyond the onset of wrinkling, however, new deformation modes may appear, with characteristics that depend sensitively on the details of the substrate response ${ }^{[32]}$. For a thin elastic film supported on a viscous substrate, wrinkles lose their periodicity and transition to localized folds once the total confinement of the thin film approaches one third of the initial wrinkling wavelength ${ }^{[33,34]}$. The transition to localized deformation may even lead to the fracture of the film ${ }^{[35]}$. For an elastomeric foundation, wrinkles lose their initial periodicity through the emergence of sub-harmonic modes due to nonlinear contributions to the elastic response of the substrate $e^{[36,37]}$. Typically, a progression from wrinkles to a period-doubled state is observed, followed by a period-quadrupled state, and finally the formation of selfcontacting folds ${ }^{[32,37-39]}$. The emergence of sub-harmonic spatial modes in this context is analogous to appearance of temporal sub-harmonics for a non-linear oscillator ${ }^{[38]}$.

In light of the key role played by the substrate in driving post-wrinkling bifurcations, it follows that this behavior should be highly sensitive to the state of the substrate, for example the presence of a compressive or tensile pre-stress. In spite of this 
expectation, however, only a limited number of studies along these lines have been conducted. In particular, Hutchinson and co-workers have predicted the emergence of localized ridges as a different type of post-wrinkling bifurcation for large substrate pretension from numerical modeling. ${ }^{[40-42]}$ The application of in-plane pre-tension causes a softening of the outward deflection of the substrate relative to the inward deflection and hence promotes the formation of ridges. Conversely, the application of in plane precompression stiffens outward deflection of the substrate relative to inward, favoring the formation of folds. While a few experiments have indicated the emergence of ridges ${ }^{[43-46]}$ or other modes of strain localization ${ }^{[42]}$ with pre-tension, the level of agreement with theory remains unclear. Further, there has been little work focused on the case of substrate pre-compression. Thus, there remains much to understand about how postwrinkling behavior depends on substrate pre-stretch.

The critical importance of a pre-stretched substrate is demonstrated by the simple experimental result shown in Figure 1. Using an approach that will be described in detail below, a soft elastomeric substrate is subjected to: (a) a tensile pre-stretch, (b) no pre-stretch, or (c) a compressive pre-stretch, prior to formation of the stiff skin. At the same applied nominal film strain, the resulting morphologies vary dramatically. With substrate pre-tension, the surface remains uniformly wrinkled; with no pre-strain, it shows the clear emergence of a sub-harmonic (period-doubled) mode; and with precompression, it shows a highly irregular 'chaotic' pattern. The aim of this study is to establish, through a combination of experiments and simulations, how substrate prestretch alters the onset and evolution of post-wrinkling modes as well as to understand the chaotic emergence of different sub-harmonic modes by adopting a general method to study the symmetry breaking from a periodic structure. 


\section{Results}

Our experiments rely on an elastic trilayer system modified from a recently described approach (Figure 2) ${ }^{[47]}$. A 400- $\mu$ m-thick soft (shear modulus $\mu_{\mathrm{s}}=12.5 \mathrm{kPa}$ ) poly(dimethylsiloxane) (PDMS) substrate is attached to a 1 -mm-thick stiffer $(\mu=260$ $\mathrm{kPa}$ ) PDMS mounting layer that has been uniaxially stretched to an initial length $L_{0}$. This bilayer is then partially released, or stretched further, to a new length $L$, placing the substrate under a pre-stretch $\lambda_{0}=L / L_{0}$. The importance of the mounting layer is that it allows for the substrate to be loaded under pre-compression without undergoing macroscopic buckling. Next, the surface is exposed to UV/ozone, which converts the top layer of the PDMS substrate into a gradient-modulus silicate film with an effective film thickness of 3-8 $\mu \mathrm{m}$ that shows excellent adhesion to the substrate. ${ }^{[48]}$. We consider the film to be stress free at this point, although we note the possibility for a slight residual stress due to mismatches in thermal expansion and/or oxidation-induced changes in volume. A subsequent partial release of the mounting layer to length $l$ now places the bilayer under compression, which we characterize in terms of the nominal strain applied to the film $\varepsilon_{\mathrm{f}}=(L-l) / L$. The subsequent wrinkling and post-wrinkling behavior of the surface as a function of applied strain is then characterized by in situ optical profilometry. Using a defect in the wrinkling pattern as a point of reference, we track the same region while increasing the compressive strain in the film.

We plot measured amplitudes for several different levels of pre-strain $\left(\lambda_{0}=0.7-\right.$ 1.2) in Figure 3 (black triangles). The amplitude associated with a given feature on the surface is defined as half of the average out-of-plane displacement between a given 
'trough' and its two neighboring 'crests'. For each sample, at least seven features are measured. The critical strain for wrinkling varied for different samples between 0.03-0.05, while the wrinkle wavelength was $l_{\mathrm{w}}=100-160 \mu \mathrm{m}$. This degree of variability is apparently due to slight differences in the position of the sample relative to the UV light source during oxidation. Using the approach of Stafford and co-workers ${ }^{[49]}$, the effective thicknesses of the silicate layers are estimated for each sample with no substrate prestretch as $h_{\mathrm{f}}=3-8 \mu \mathrm{m}$, with shear moduli of $\mu_{\mathrm{f}}=620 \mathrm{kPa}-3.8 \mathrm{MPa}$, corresponding to modulus mismatch values $\left(\mu_{\mathrm{f}} / \mu_{\mathrm{s}}\right)$ of $50-300$. These large ranges in effective film thickness and modulus are the result of variations between different samples. However, as shown by Cao and Hutchinson, the modulus mismatch does not affect the critical strain for post-wrinkling bifurcations if the mismatch is above $10 .^{[41]}$

In the wrinkle regime, the amplitudes are nearly homogeneous, as can be seen from the small amount of scatter in Figure 3 (standard deviations in wrinkle amplitudes are typically less than $1 \mu \mathrm{m}$ ), and grow with increasing strain. However, at a sufficiently large value of $\varepsilon_{\mathrm{f}}$, a post-wrinkling bifurcation is observed in which some wrinkles grow in amplitude while their neighbors shrink. As seen in Figure 3(a-c), pre-stretches from $\lambda_{0}=$ $1.0-1.2$ show a clean bifurcation corresponding to a period doubled state, as first shown by Brau, et al. ${ }^{[38]}$ for the case of $\lambda_{0}=1$. We denote the strain at which this first postwrinkling bifurcation is observed as $\varepsilon_{\mathrm{pw}}$, identified in practice as the point at which the standard deviation of wrinkle amplitudes show a dramatic increase. For the case of $\lambda_{0}=1$, we verified that the period doubling bifurcation showed no apparent hysteresis from initial loading, to unloading, and subsequent reloading (Supporting Information, Figure $\mathrm{S} 1$ ), suggesting that plastic deformation plays little if any role in this process. 
For $\lambda_{0}=1$ (Figure $3 \mathrm{c}$ ), we measure a value of $\varepsilon_{\mathrm{pw}}=0.18$, which is in good agreement with the values reported by Brau, et al. of $0.17-0.20$ for a true bilayer system ${ }^{[38]}$. When the substrate is under slight pre-tension, the period-doubled bifurcation shifts to higher strains but remains otherwise qualitatively similar; at $\lambda_{0}=1.2$ we measure $\varepsilon_{\mathrm{pw}}=$ 0.23 for the sample characterized in Figure $3 \mathrm{a}$ and at $\lambda_{0}=1.1$ we measure $\varepsilon_{\mathrm{pw}}=0.22$ for the sample in Figure $3 \mathrm{~b}$. Under slight pre-compression the bifurcation occurs at lower film strain; at $\lambda_{0}=0.9$ and $\lambda_{0}=0.8$ (Figure $3 \mathrm{~d}-\mathrm{e}$ ), we find $\varepsilon_{\mathrm{pw}}=0.11$ and $\varepsilon_{\mathrm{pw}}=0.10$, respectively. While a clear bifurcation into a bimodal collection of larger and smaller amplitudes can still be seen in this case, consistent with period doubling, the spread in amplitudes for each population is larger than for $\lambda_{0}=1$ and $\lambda_{0}=1.2$.

This non-uniformity becomes dramatically more pronounced for a film on a substrate with a larger pre-compression, $\lambda_{0}=0.7$ (Figure 3f). In this case, a clean bifurcation is lost, replaced instead by the emergence of a broad spread of amplitudes at a strain of $\varepsilon_{\mathrm{pw}}=0.06$, which is only slightly above the critical strain for wrinkling, $\varepsilon_{\mathrm{w}}=$ 0.04. The transition from regular wrinkles to irregular, or 'chaotic', structures occur over such a small range of strain that when plotted on the same scale as the other samples in Figure 3a-e, the distinction between wrinkling and post-wrinkling is completely obscured; however, the inset to Figure $3 \mathrm{f}$ shows that indeed a narrow range of regular wrinkling can be discerned.

To better understand these effects, we model the system using the commercial finite element software ABAQUS. Both the film and substrate are taken to be incompressible neo-Hookean materials. For a ratio of the film to substrate modulus of only $50-300$, as studied here, the maximum strain in the film can be as large as $\sim 0.20$ in 
our simulations. Thus, it is necessary to use a non-linear hyperelastic model for both the film and substrate. However, as the range of strains is still modest, the neo-Hookean model should provide a reasonable description of both materials. The substrate is uniaxially pre-stretched while the film/substrate bilayer is subsequently compressed under plane strain, which is similar to the condition in the experiments. To simulate the period doubled state, we choose a unit cell of width $l_{w}$, the wrinkle wavelength. Symmetry is maintained across one boundary and uniform strain is applied on the other boundary. A small amplitude initial imperfection is introduced to break the translational symmetry of the surface. More details about the simulations of the period doubled state will be discussed below. When compressive strain is incrementally applied, the surface initially remains flat and then forms wrinkles after a critical strain. With further increase in strain, the wrinkle amplitude grows but the nominal wavelength does not change. After a second critical strain, wrinkles lose their original periodicity through a supercritical period-doubling bifurcation. We record the second critical strain as $\varepsilon_{\mathrm{pw}}$.

In Figure 3, we plot the simulation results for wrinkle amplitudes with different substrate pre-stretches as red lines. From the experimentally measured wrinkle wavelength, and the dependence of wrinkle amplitude on strain, we estimate the film thickness by fitting the classical buckling equations ${ }^{[49]}$, as before. For the effective film modulus, however, we make use of the theoretical prediction that takes into account substrate pre-stretch and assumes infinite substrate thickness ${ }^{[4]}$. The modulus ratios of the film to substrate estimated in this fashion are (a) 120, (b) 200, (c) 50, (d) 130, (e) 225 and (f) 35, while the film thickness are (a) $5.8 \mu \mathrm{m}$, (b) $6.0 \mu \mathrm{m}$, (c) $8.0 \mu \mathrm{m}$, (d) $6.6 \mu \mathrm{m}$, (e) $5.5 \mu \mathrm{m}$ and (f) $7.8 \mu \mathrm{m}$. In the simulations, we choose a modulus ratio to match the 
experimental value for each plot. The ratio of the total thickness to the film thickness is fixed at a representative value 50 in all cases, since the ratio has no effect on the wrinkling and post-wrinkling as long as the substrate is much thicker than the film, and can be treated as infinite. As seen in Figure 3, the simulations generally agree well with the experimental results, in terms of both the location of the post-wrinkling bifurcation and the subsequent evolution of amplitudes. The agreement of the cases with pre-tension in the substrate is slightly better than with pre-compression. A possible reason is the competition between different post-wrinkling bifurcation modes, which clearly influences the behavior at large pre-compression, as we discuss further below.

Figure 4 shows the dependence of $\varepsilon_{\mathrm{pw}}$ on $\lambda_{0}$, obtained from finite element simulations and experiments on at least 3 different samples at each $\lambda_{0}$. In this simulation and the remainder of the paper, the ratio of the film modulus to substrate modulus is fixed as 50 , since $\varepsilon_{\mathrm{pw}}$ is almost independent of the modulus ratio as long as the modulus ratio is larger than $10^{[41]}$. The ratio of the total thickness to the film thickness is also fixed as 50 , which is large enough for the thickness of the substrate to be considered as infinite. When there is no pre-stretch in the substrate, $\varepsilon_{\mathrm{pw}}$ is calculated to be around 0.18 , which matches the experimental value of $0.18 \pm 0.02$. A monotonic increase in $\varepsilon_{\mathrm{pw}}$ with $\lambda_{0}$ is seen: precompression in the substrate decreases $\varepsilon_{\mathrm{pw}}$, while pre-tension increases $\varepsilon_{\mathrm{pw}}$. Under pretension, e.g. $\lambda_{0}=1.2$, the finite element result of $\varepsilon_{\mathrm{pw}}=0.26$ agrees well with that from experiments $(0.27 \pm 0.07)$. Under pre-compression, e.g. at $\lambda_{0}=0.7$, the respective experimental and simulation results of $\varepsilon_{\mathrm{pw}}=0.05 \pm 0.02$ and 0.06 are again in agreement. Overall, the simulations in Figures 3 and 4 show almost quantitative agreement with 
experimental data, and the qualitative trend of $\varepsilon_{\mathrm{pw}}$ monotonically and strongly increasing with $\lambda_{0}$ over this range of substrate pre-stretch is clear in both cases.

We next consider the irregularity of amplitudes observed during post-wrinkling bifurcation for substrates under pre-compression. In Figure 5, we compare in detail the evolution of surface topography with increasing film strain for samples with $\lambda_{0}=1.0$ and 0.7. Color maps of the surface profiles are shown over large areas of the sample (Figure 5a-c, e-g), along with line profiles (Figure $5 \mathrm{~d}, \mathrm{~h}$ ) at the locations indicated. The profiles are taken at nearly the same position on each sample, and plotted with an $\mathrm{x}$-axis normalized by $\left(1-\varepsilon_{f}\right)$ to vertically align each crest and trough so that its evolution with film strain can be easily observed.

With no pre-stretch, the sample evolves from the wrinkled state (green trace in Figure $5 \mathrm{~d}$ ) at $\varepsilon_{\mathrm{f}}=0.083$ to a period-doubled state at $\varepsilon_{\mathrm{f}}=0.16$ (blue trace) and a periodquadrupled state at $\varepsilon_{\mathrm{f}}=0.23$ (red trace). The amplitudes evolve in a fairly regular fashion across the entire sample, except in regions close to a defect in the wrinkling pattern (i.e., as seen to the lower left of Figures 5a-c and correspondingly to the left side of the traces in Figure 5d).

With a pre-compression of $\lambda_{0}=0.7$, however, dramatically different behavior is observed. A regular wrinkle pattern is first observed, as seen for $\varepsilon_{\mathrm{f}}=0.045$ (Figure 5e, green trace in Figure 5h), but rapidly evolves into a set of deep features with irregular spacing by $\varepsilon_{\mathrm{f}}=0.11$ (Figure $5 \mathrm{~g}$, red trace in Figure $5 \mathrm{~h}$ ). Examination of the behavior at intermediate strains, e.g. at $\varepsilon_{\mathrm{f}}=0.063$ suggests that this pattern emerges through the competition between multiple different sub-harmonic modes with periodicities of 2, 3, 
and 4 times the fundamental wrinkle mode. In other words, some regions show behavior locally characteristic of period doubling (two troughs that grow in depth separated by one that becomes more shallow), while others show characteristics of period tripling (deep troughs separated by two shallow troughs) and quadrupling (separation by three shallow troughs).

Similar behavior can be observed by using laser scanning confocal fluorescence microscopy to characterize samples with fluorescently labeled substrates. With no substrate pre-stretch (Figure 6a), regular wrinkles first develop at $\varepsilon_{\mathrm{f}}=0.04$, then grow in amplitude before period doubling takes place at $\varepsilon_{\mathrm{f}}=0.17$ When the substrate is under a small amount of pre-compression, $\lambda_{0}=0.9$ (Figure 6b), wrinkles first give way to a shallow period doubled state at $\varepsilon_{\mathrm{f}}=0.14$, and by $\varepsilon_{\mathrm{f}}=0.17$, the sample is well past the onset of period doubling, with the amplitude of the diminishing wrinkles approaching zero. By $\varepsilon_{\mathrm{f}}=0.22$, the bilayer with $\lambda_{0}=0.9$ has already transitioned to the period quadrupled state; whereas for $\lambda_{0}=1.0$, the sample remains in the period doubled state even at a slightly higher film strain of 0.24 . With larger pre-compression, $\lambda_{0}=0.7$ (Figure 6c), wrinkles form but are soon followed by a 'chaotic' surface pattern with shallow regions of the period doubled, period tripled and period quadrupled states. Interestingly, this behavior shows variability across the transverse direction of the sample, as seen in Figure $5 \mathrm{~g}$ and Video $\mathrm{S} 1$. We suspect this behavior arises because different regions of the sample spontaneously break the symmetry of the wrinkle pattern in different ways, leading to the presence of 'domain boundaries' where one set of deep features meets another, mismatched set. However, further study is required to better understand this process. 
The emergence of such chaotic behavior can be anticipated based on the analogy to the nonlinear oscillator, ${ }^{[38,50-52]}$ which is well-known to show chaotic behaviors in certain regimes. However, to provide a better understanding of the driving force in the current system, we use finite element methods to compare the energies of the different sub-harmonic modes. To enable such comparisons at equivalent levels of compression, we employ simulation cells with several different sizes, boundary conditions, and initial imperfections, to enforce different symmetries on the post-wrinkled states. For example, Figure 7 shows simulated surface height profiles for different modes at a constant film strain of $\varepsilon_{\mathrm{f}}=0.11$ and pre-stretch of $\lambda_{0}=0.7$ : wrinkling that maintains the initial periodicity (Figure 7a), period doubling (Figure 7b and Video S2), low-energy tripling (Figure 7c and Video S3), high-energy tripling (Figure 7d) and period quadrupling (Figure 7e and Video S4).

To enforce the initial wrinkle period to be conserved throughout the application of the strain, as in Figure $7 \mathrm{a}$, we simulate a unit cell with half the nominal wrinkle wavelength $l_{w} / 2$. One boundary of the unit cell is chosen at a trough of the wrinkles and the other is chosen at the neighboring peak. We apply reflection symmetry on one boundary and uniform strain on the other boundary, so that both boundaries are kept flat. The corresponding energy per wavelength $l_{w}$ is calculated and called $U_{l}$.

To study the bifurcation from a periodic wrinkled state to a period-doubled state (Figure 7b), we use a unit cell of width $l_{w}$. We can position the boundaries of the unit cell in two ways: at the troughs of two neighboring wrinkles (Figure 8b), or at the peaks (Figure $8 \mathrm{c})$. The simulations start with a nearly flat surface with small amplitude (1/2000 times the film thickness) prescribed imperfections, shown in Figure $8 \mathrm{~b}$ and $\mathrm{c}$ greatly 
magnified in amplitude for visual clarity. In both cases, symmetry condition is applied on one boundary and uniform strain is applied on the other boundary. Our simulations indicate that the first choice of boundaries (Figure 8b) leads to period-doubling at the critical strain $\varepsilon_{\mathrm{pw}}$, and we call the energy per wavelength $l_{w}$ of this configuration $U_{2}$. By contrast, for the second choice of boundaries, the system retains the initial wrinkle periodicity and does not undergo period doubling.

To study the bifurcation to a period-tripled state (Figure $7 \mathrm{c}$ and d), we use a unit cell of width $3 l_{w} / 2$, which allows for period-tripling but prevents period-doubling. There is only one choice for placing the boundaries of the unit cell, with one boundary at a trough and the other boundary at a peak of the wrinkles (Figure 9a). Again, we apply symmetry condition and uniform strain to the two boundaries. The period-tripled state can lead to two modes (Figure 7c and d), which are selected through the initially prescribed small amplitude imperfections, as shown in Figure $9 \mathrm{~b}$ and 9c. In one mode of period tripling, of the three neighboring troughs, one becomes deeper and the other two become shallower (Figure $7 \mathrm{c}$ and $9 \mathrm{~b}$ ). In the other mode of period tripling, of the neighboring troughs, one becomes shallower and the other two become deeper (Figure 7d and $9 \mathrm{c})$. We call the energy per wavelength $l_{w}$ of both modes $U_{3}$. Figure $9 \mathrm{~d}$ plots the difference in the energies between the two tripling modes and the doubling mode, $U_{3}-U_{2}$, as a function of the film strain for a pre-stretch of $\lambda_{0}=0.7$. The mode in Figure $9 \mathrm{~b}$ has a lower energy than the mode in Figure 9c, and thus for the remainder of the paper, we only consider the former, lower energy, mode of period tripling. Remarkably, this lowenergy tripling mode has a lower energy than the period-doubling mode at sufficiently large film strains - a point to which we will return below. 
To study the bifurcation to a period quadrupled state (Figure 7e), we use a unit cell of width $2 l_{w}$, which allows both period doubling and quadrupling. Similar to period doubling, we can choose the boundaries of the unit cell in two ways: at two troughs (Figure 10b) or at two peaks (Figure 10c). Moreover, similar to period tripling, there can be more than one mode of the period-quadrupled states even when the boundary of the unit cell is chosen. Here, we will not go into details regarding these different modes, but instead focus on the mode of quadrupling corresponding to that observed in experiments (Figure 7e), which is selected by the prescribed imperfections shown in Figure 10b. In this case the system first undergoes period doubling, followed by period quadrupling, and we denote the energy per wavelength $l_{w}$ as $U_{4}$.

In Figure 11a-d, we plot the normalized energy by which each of the configurations $U_{2}, U_{3}, U_{4}$ differ from the wrinkled state $U_{1}$, as a function of the applied strain $\varepsilon_{\mathrm{f}}$. We normalize the energies by $\mu_{\mathrm{s}} l_{\mathrm{w}} h_{\mathrm{f}}$, where $h_{\mathrm{f}}$ is the thickness of the film. When a given curve falls below 0 , the corresponding type of period multiplication is energetically favorable, with the lowest energy curve representing the ground state. As seen in Figure 11a for no pre-stretch, the system remains in the wrinkled state and thus all of the curves stay at an energy of 0 , until a strain of $\varepsilon_{\mathrm{f}}=0.19$. Beyond this point, the curves for $U_{2}$ and $U_{4}$ become negative, corresponding to the period-doubled state. At a strain of $\varepsilon_{\mathrm{f}}=0.25$, the curve for $U_{4}$ becomes lower in energy than $U_{2}$, corresponding to the period-quadrupled state. Over this range of strain, the energy $U_{3}$ remains above both $U_{2}$ and $U_{4}$, and thus period tripling is energetically disfavored. These findings are in excellent agreement with our experiments at $\lambda_{0}=1.0$, as well as previous work, showing clean period doubling and period quadrupling bifurcations ${ }^{[32,37-39]}$. 
The pre-tensioned substrate stabilizes both the wrinkled and period-doubled states, as seen in Figure $11 \mathrm{~b}$ for $\lambda_{0}=1.2$. Here, the onset of period doubling is delayed until $\varepsilon_{\mathrm{f}}=$ 0.25 , while over the range of strain studied (up to $\varepsilon_{\mathrm{f}}=0.32$ ) period quadrupling is not observed at all, and $U_{3}$ remains well above $U_{2}$. The simulation results show excellent agreement with our experimental findings that period doubling is shifted to higher strains when there is pre-tension in the substrate and no period quadrupling is observed over the range of $\varepsilon_{\mathrm{f}}$ studied.

In contrast, the pre-compressed substrate de-stabilizes both the wrinkled and period-doubled states compared to the period-tripled and quadrupled states (Figure 11c and d). As seen in Figure 11c for $\lambda_{0}=0.85$, the curve for $U_{2}$ falls below zero at a strain of 0.13 , only slightly before $U_{3}$ does, at 0.15 . The period-tripled state $U_{3}$ then falls below $U_{2}$ and $U_{4}$ at a strain of 0.18 . As seen in Figure $11 \mathrm{~d}$ for $\lambda_{0}=0.7$, the curves for $U_{2}$ and $U_{3}$ fall below zero at very similar strains $\left(\varepsilon_{\mathrm{f}}=0.07-0.08\right)$. The period-quadrupled state $U_{4}$ falls below $U_{2}$ at only slightly higher strain $\left(\varepsilon_{\mathrm{f}}=0.10\right)$, and remains very close to $U_{3}$ for larger strains. Because period doubling, tripling and quadrupling all happen at strains close to the formation of wrinkles, wrinkles are still very shallow, and the energies of period doubling, tripling and quadrupling modes in the range of $\varepsilon_{\mathrm{f}}=0.07-0.10$ are very close to each other. The inset magnifies the energies in this region of strain.

We further illustrate the effect of pre-stretch by plotting the difference between $U_{3}$ and $U_{2}$ for several values of $\lambda_{0}$ (Figure 12). The pre-compressed substrate greatly reduces the energy difference between the period-tripled state and the period-doubled state. Presumably, this similarity in energy means that the presence of defects or even slight 
spatial variations in material properties would lead to local biases in favor of a given subharmonic mode, thus yielding a globally chaotic behavior. The prediction from the simulation is qualitatively in very good agreement with our experimental observations.

\section{Conclusion}

Our study reveals the strong effect of substrate pre-stretch on post-wrinkling bifurcations. Pre-stretch shifts the critical strain for period doubling: modest amounts of pre-tension are found to stabilize wrinkles to higher strains, while modest precompression promotes the emergence of sub-harmonic modes at lower strains. For large substrate pre-compression, a new type of spatially chaotic behavior is found. A newly developed computational approach allows for the study of symmetry breaking from a periodic structure by the emergence of different sub-harmonic modes through the prescription of different simulation cell sizes, initial imperfections, and boundary conditions. The computational results reveal that energies of the different subharmonic modes become similar when the substrate is pre-compressed, thus eliminating the preference for a clean sequence of period doubling and quadrupling bifurcations. We anticipate that substrate pre-stretch will be an important parameter in future efforts to control the morphologies of wrinkled and post-wrinkled surfaces.

\section{Methods}

A trilayer system was used to independently vary the compression applied to the substrate and the silicate film. A silica reinforced vinyl-terminated polydimethylsiloxane (PDMS) mounting layer (DMS-V31S15, Gelest Inc.) with a shear modulus $260 \mathrm{kPa}$ and thickness $1 \mathrm{~mm}$ was uniaxially pre-stretched on a stretcher. A pre-cured substrate with 
thickness of approximately $400 \mu \mathrm{m}$ composed of a PDMS elastomer (Sylgard 184, Dow Corning) with a 50:1 weight ratio of base to crosslinker (shear modulus $12.5 \mathrm{kPa}$ based on dynamic mechanical analysis) was attached to the pre-stretched mounting layer using a thin (2-5 $\mu \mathrm{m}$ thick) layer of uncured PDMS with the same composition as the substrate. This bilayer assembly was placed in an oven at $60{ }^{\circ} \mathrm{C}$ for $4 \mathrm{~h}$ to bond the layers, and then allowed to cool to room temperature over $30 \mathrm{~min}$ before deforming the mounting layer to put the substrate under a pre-stretch $\lambda_{0}$. The substrate was left at $\lambda_{0}$ for $30 \mathrm{~min}$ before being placed in a $\mathrm{UV} / \mathrm{O}_{3}$ cleaner for 25 min to convert the surface of the PDMS substrate into a stiffer oxide layer. For each sample, we determined the amplitudes and spacings between at least seven neighboring wrinkles from optical profilometry. The oxide films were found to have effective thicknesses of 3-8 $\mu \mathrm{m}$ and shear moduli of $620 \mathrm{kPa}-3.8$ $\mathrm{MPa}$ as determined by fitting the dependence of the wrinkle amplitude and wavelength on film strain for samples with no substrate pre-stretch via the classical buckling equations as described by Stafford and others ${ }^{[12,16]}$. For any given sample, the respective uncertainties in these quantities were less than $0.6 \mu \mathrm{m}$ and $50 \mathrm{kPa}$; the large ranges determined for different samples therefore presumably reflect slight differences in sample position within the $\mathrm{UV} / \mathrm{O}_{3}$ cleaner. However, as the film modulus is larger than the substrate modulus by a factor of 50 - 300, this variation should not play a significant role in the observed behavior. Compression was applied to the trilayer by relaxing the prestretch of the mounting layer.

Optical profilometry (Zygo NewView 7300) was used for in-situ characterization of the height profiles of the sample surfaces using MetroPro 8.3.2 software. The $5 \mathrm{x}$ and 10x lenses were utilized as well as the stitch application to characterize a large area of the 
sample. At high film strains only the data points from the crest and trough could be imaged due to the steep angles of the surface; a spline was used to fill in the missing segments of the surface profiles in Figure 1 and Figure 5. Laser scanning confocal fluorescence microscopy (Zeiss LSM 510 Meta) was used to characterize the shape of the wrinkles. Fluorescent labeling of the substrate was accomplished by adding $4.5 \mu \mathrm{g}$ of fluorescein-o-acrylate in 1,4-dioxane per $1 \mathrm{~g}$ of PDMS prior to curing. During imaging, a refractive index matching fluid consisting of $72 \%$ glycerol and $28 \%$ water (weight percent) was used along with a $25 \mathrm{x}$ variable immersion lens. Several two-dimensional frames were taken and manually stitched together by aligning peaks and troughs of the wrinkles to cover a larger area of the sample.

The commercial finite element software ABAQUS was used to computationally study the effect of pre-stretch on post-wrinkling bifurcations. The substrate was uniaxially pre-stretched and then the film-substrate bi-layer compressed under plane strain. Both the film and substrate were modeled as incompressible neo-Hookean materials, and the free energy of neo-Hookean material with a pre-stretch was implemented by a user-defined subroutine UMAT. For all the simulations except Figure 3 , the modulus ratio of the film to substrate was set to 50 , and the thickness ratio of the bilayer to the film was chosen as 50 , both of which roughly match the experimental conditions. To break the symmetry of the surface during compression, the first and second eigenmodes obtained by a linear perturbation step were introduced as initial imperfections with very small amplitudes (roughly $1 / 2000$ times the film thickness).

We call the wrinkle wavelength $l_{w}$. When we simulate the critical strain for period doubling (Figures 3 and 4), the simulation sample length is chosen as $l_{w}$. To understand 
chaotic period multiplication (Figures 7-12), we calculate the energy of different periodic states: wrinkling with the initial periodicity, period doubling, tripling and quadrupling. To realize these different periodicities, we simulate samples with lengths $l_{w} / 2, l_{w}, 3 l_{w} / 2$ and $2 l_{w}$ respectively. Symmetry condition is added on one of the boundary and uniform strain is applied on the other boundary. Static method is used for all the simulations .

Acknowledgement. The work at UMass was supported by NSF grant DMR-1309331, with additional support by the NSF MRSEC (DMR-0820506). AA was supported by an NSF Graduate Research Fellowship (DGE-0907995). The work at Harvard was supported by the NSF MRSEC (DMR-0820484). 


\section{References}

[1] E. Cerda, L. Mahadevan, Phys. Rev. Lett. 2003, 90, 074302.

[2] J. Genzer, J. Groenewold, Soft Matter 2006, 2, 310.

[3] P.-C. Lin, S. Yang, Soft Matter 2009, 5, 1011.

[4] J. Y. Chung, J. P. Youngblood, C. M. Stafford, Soft Matter 2007, 3, 1163.

[5] D. Xia, L. M. Johnson, G. P. López, Adv. Mater. 2012, 24, 1287.

[6] S. G. Lee, D. Y. Lee, H. S. Lim, D. H. Lee, S. Lee, K. Cho, Adv. Mater. 2010, 22, 5013.

[7] P.-C. Lin, S. Vajpayee, A. Jagota, C.-Y. Hui, S. Yang, Soft Matter 2008, 4, 1830.

[8] S. Yang, K. Khare, P.-C. Lin, Adv. Funct. Mater. 2010, 20, 2550.

[9] E. P. Chan, E. J. Smith, R. C. Hayward, A. J. Crosby, Adv. Mater. 2008, 20, 711.

[10] P. Kim, Y. Hu, J. Alvarenga, M. Kolle, Z. Suo, J. Aizenberg, Adv. Opt. Mater. 2013, 1,381 .

[11] E. Lee, M. Zhang, Y. Cho, Y. Cui, J. Van der Spiegel, N. Engheta, S. Yang, Adv. Mater. 2014, 1.

[12] J. Y. Chung, A. J. Nolte, C. M. Stafford, Adv. Mater. 2011, 23, 349.

[13] E. A. Wilder, S. Guo, S. Lin-Gibson, M. J. Fasolka, C. M. Stafford, Macromolecules 2006, 39, 5956.

[14] D.-Y. Khang, J. A. Rogers, H. H. Lee, Adv. Funct. Mater. 2009, 19, 1526.

[15] J. Huang, M. Juszkiewicz, W. H. de Jeu, E. Cerda, T. Emrick, N. Menon, T. P. Russell, Science 2007, 317, 650.

[16] C. M. Stafford, C. Harrison, K. L. Beers, A. Karim, E. J. Amis, M. R. VanLandingham, H.-C. Kim, W. Volksen, R. D. Miller, E. E. Simonyi, Nat. Mater. 2004, 3, 545 .

[17] J. A. Howarter, C. M. Stafford, Soft Matter 2010, 6, 5661.

[18] C. Lu, H. Möhwald, A. Fery, Soft Matter 2007, 3, 1530. 
[19] A. Schweikart, A. Fery, Microchim. Acta 2009, 165, 249.

[20] A. Chen, D. K. Lieu, L. Freschauf, V. Lew, H. Sharma, J. Wang, D. Nguyen, I. Karakikes, R. J. Hajjar, A. Gopinathan, E. Botvinick, C. C. Fowlkes, R. a Li, M. Khine, Adv. Mater. 2011, 23, 5785.

[21] S. Chung, J. H. Lee, M.-W. Moon, J. Han, R. D. Kamm, Adv. Mater. 2008, 20, 3011 .

[22] K. Khare, J. Zhou, S. Yang, Langmuir 2009, 25, 12794.

[23] Y. Mei, D. J. Thurmer, F. Cavallo, S. Kiravittaya, O. G. Schmidt, Adv. Mater. 2007, 19, 2124.

[24] J. B. Kim, P. Kim, N. C. Pegard, S. J. Oh, C. R. Kagan, J. W. Fleischer, H. A. Stone, Y. Loo, Nat. Photonics 2012, 6, 327.

[25] M. S. White, M. Kaltenbrunner, E. D. Głowacki, K. Gutnichenko, G. Kettlgruber, I. Graz, S. Aazou, C. Ulbricht, D. A. M. Egbe, M. C. Miron, Z. Major, M. C. Scharber, T. Sekitani, T. Someya, S. Bauer, N. S. Sariciftci, Nat. Photonics 2013, 7,811 .

[26] D. J. Lipomi, B. C.-K. Tee, M. Vosgueritchian, Z. Bao, Adv. Mater. 2011, 23, 1771 .

[27] S. Lee, S. Kim, T.-T. Kim, Y. Kim, M. Choi, S. H. Lee, J.-Y. Kim, B. Min, Adv. Mater. 2012, 24, 3491.

[28] D.-Y. Khang, H. Jiang, Y. Huang, J. a Rogers, Science 2006, 311, 208.

[29] G. M. Whitesides, Adv. Mater. 2004, 16, 1375.

[30] M. Guvendiren, J. A Burdick, Adv. Healthc. Mater. 2013, 2, 155.

[31] K. Anselme, P. Davidson, A M. Popa, M. Giazzon, M. Liley, L. Ploux, Acta Biomater. 2010, 6, 3824.

[32] F. Brau, P. Damman, H. Diamant, T. A. Witten, Soft Matter 2013, 9, 8177.

[33] L. Pocivavsek, R. Dellsy, A. Kern, S. Johnson, B. Lin, K. Y. C. Lee, E. Cerda, Science 2008, 320, 912.

[34] D. Holmes, A. Crosby, Phys. Rev. Lett. 2010, 105, 1.

[35] R. Huang, H. Yin, J. Liang, J. Sturn, K. Hobart, Z. Suo, Acta Mech. Sin. 2002, 18, 441. 
[36] B. Audoly, Phys. Rev. E 2011, 84, 011605.

[37] S. Cai, D. Breid, A. J. Crosby, Z. Suo, J. W. Hutchinson, J. Mech. Phys. Solids 2011, 59, 1094.

[38] F. Brau, H. Vandeparre, A. Sabbah, C. Poulard, A. Boudaoud, P. Damman, Nat. Phys. 2010, 7, 56.

[39] J.-Y. Sun, S. Xia, M.-W. Moon, K. H. Oh, K.-S. Kim, Proc. R. Soc. A Math. Phys. Eng. Sci. 2011, 468, 932.

[40] J. Hutchinson, Philos. Trans. R. Soc. A Math. Phys. Eng. Sci. 2013, 371.

[41] Y. Cao, J. W. Hutchinson, J. Appl. Mech. 2012, 79, 031019.

[42] J. Zang, X. Zhao, Y. Cao, J. W. Hutchinson, J. Mech. Phys. Solids 2012, 60, 1265.

[43] Y. Ebata, A. B. Croll, A. J. Crosby, Soft Matter 2012, 8, 9086.

[44] Q. Wang, X. Zhao, J. Appl. Mech. 2013, 81, DOI 10.1115/1.4025828.

[45] C. Cao, H. F. Chan, J. Zang, K. W. Leong, X. Zhao, Adv. Mater. 2013, DOI 10.1002/adma.201304589.

[46] A. Takei, L. Jin, J. W. Hutchinson, H. Fujita, Adv. Mater. 2014, 1.

[47] D. Chen, L. Jin, Z. Suo, R. C. Hayward, Mater. Horizons 2014, DOI 10.1039/c3mh00107e.

[48] J. Song, D. Tranchida, G. J. Vancso, Macromolecules 2008, 41, 6757.

[49] C. M. Stafford, C. Harrison, K. L. Beers, A. Karim, E. J. Amis, M. R. VanLandingham, H.-C. Kim, W. Volksen, R. D. Miller, E. E. Simonyi, Nat. Mater. 2004, 3, 545 .

[50] R. M. May, Nature 1976, 261, 459.

[51] J. Testa, J. Perez, C. Jeffries, Phys. Rev. Lett. 1982, 48, 714.

[52] R. C. Hilborn, Chaos and Nonlinear Dynamics: An Introduction for Scientist and Engineers, Oxford University Press, 2000. 

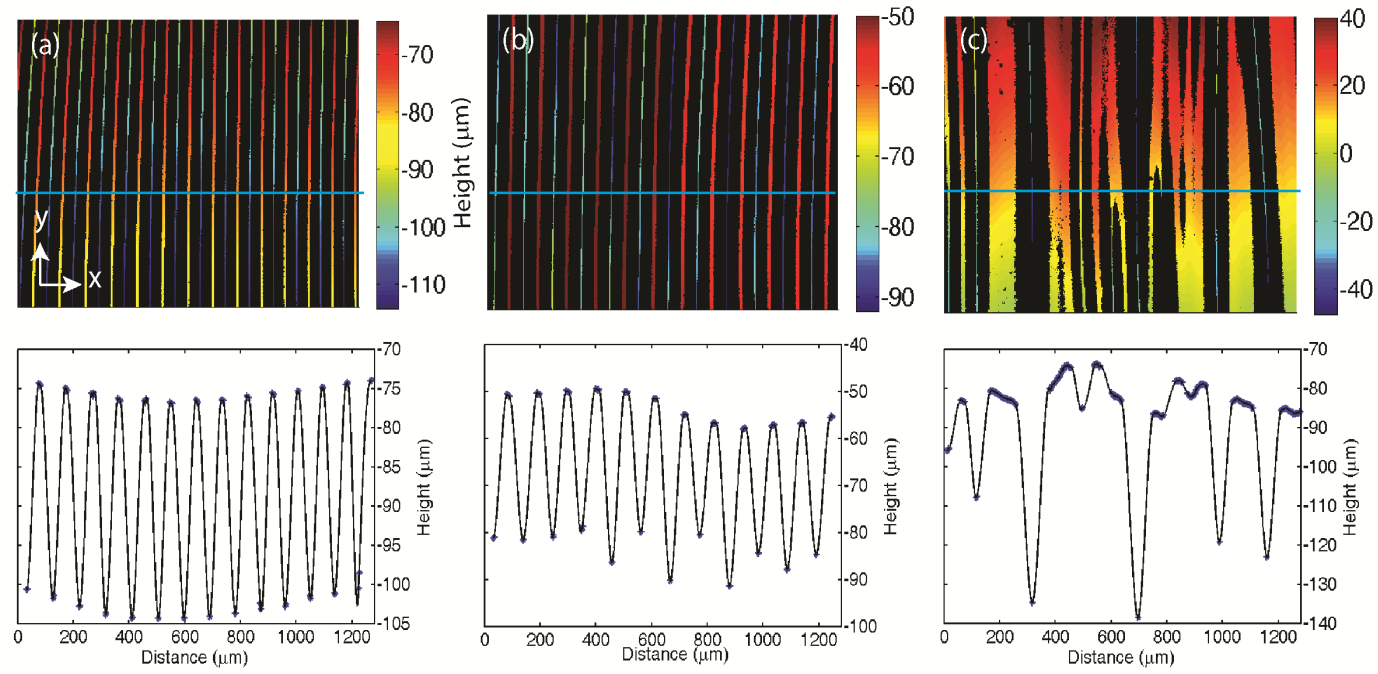

Figure 1. Optical profilometry is used to characterize the surface profiles of films at similar nominal strains $\varepsilon_{\mathrm{f}}$ on substrates with varying amounts of pre-stretch $\lambda_{0}$. (a) At $\varepsilon_{\mathrm{f}}=$ 0.16 and $\lambda_{0}=1.3$, uniform wrinkles are observed. (b) At $\varepsilon_{\mathrm{f}}=0.17$ and $\lambda_{0}=1.0$, period doubling has just begun to emerge. (c) At $\varepsilon_{\mathrm{f}}=0.15$ and $\lambda_{0}=0.7$, 'chaotic' post-wrinkling is found. 


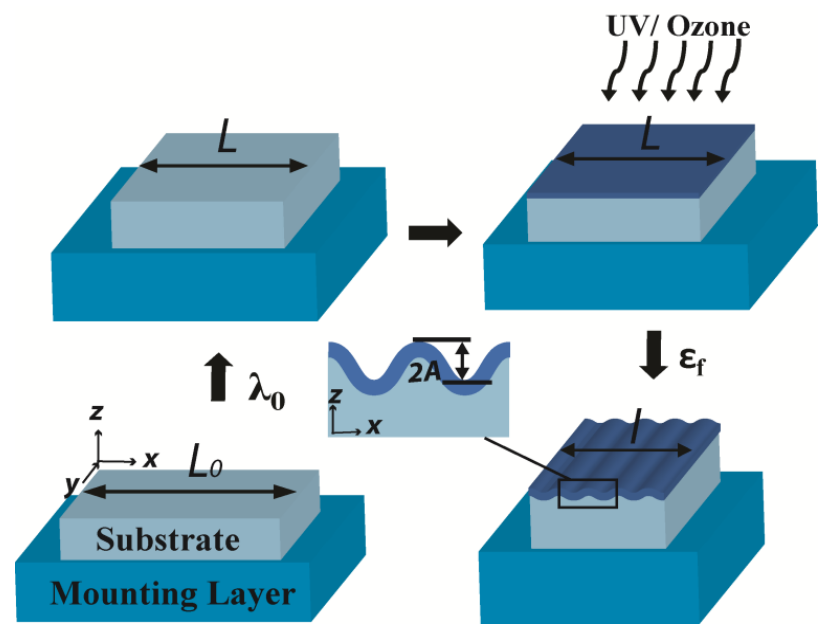

Figure 2. A schematic of the experimental procedure. An elastomeric substrate is attached to a pre-stretched mounting layer, deformed to $\lambda_{0}$, and exposed to $\mathrm{UV} / \mathrm{O}_{3}$ to form a stiff film layer. The stiff film is compressed with strain $\varepsilon_{\mathrm{f}}$ by partially releasing the mounting layer. 

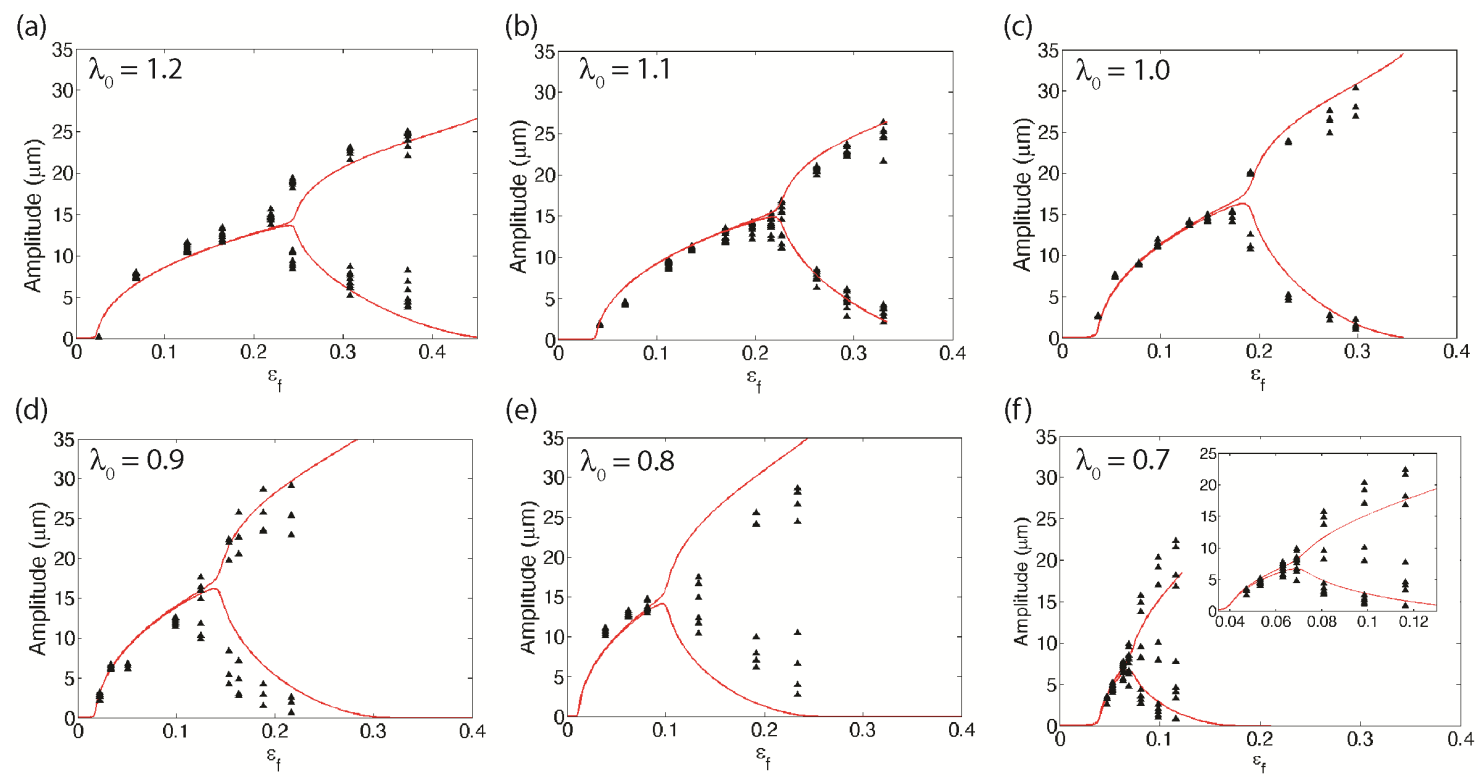

Figure 3. Bifurcation curves of the wrinkle amplitude as a function of film strain for substrates pre-stretched to $\lambda_{0}=$ (a) 1.2 , (b) 1.1 , (c) 1.0 , (d) 0.9 , (e) 0.8 and (f) 0.7 . The experimental data (black triangles) reveal a gradual shift of the bifurcation point as well as an increase in distribution of amplitudes past $\varepsilon_{\mathrm{pw}}$ with a decrease in $\lambda_{0}$. Simulations (red lines) show good agreement with the experimental data. 


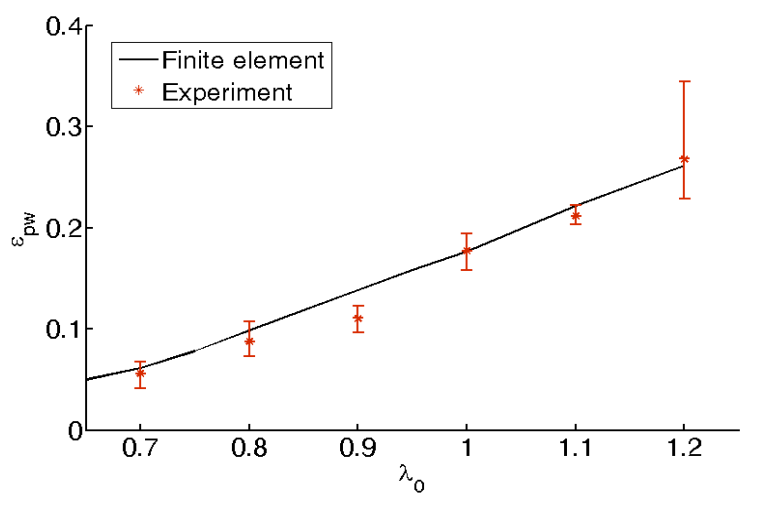

Figure 4. The critical film strain for post-wrinkling bifurcation $\varepsilon_{\mathrm{pw}}$ is plotted against the substrate pre-stretch $\lambda_{0}$. The solid lines represent finite element results and the solid symbol represents experimental results, with the error bars corresponding to minimum/maximum values for at least 3 samples. As pre-stretch increases, the film strain required for post-wrinkling bifurcation increases. 

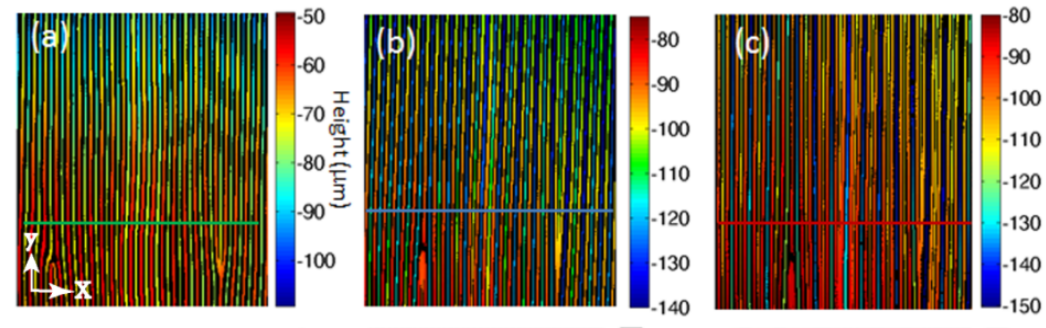

(d)
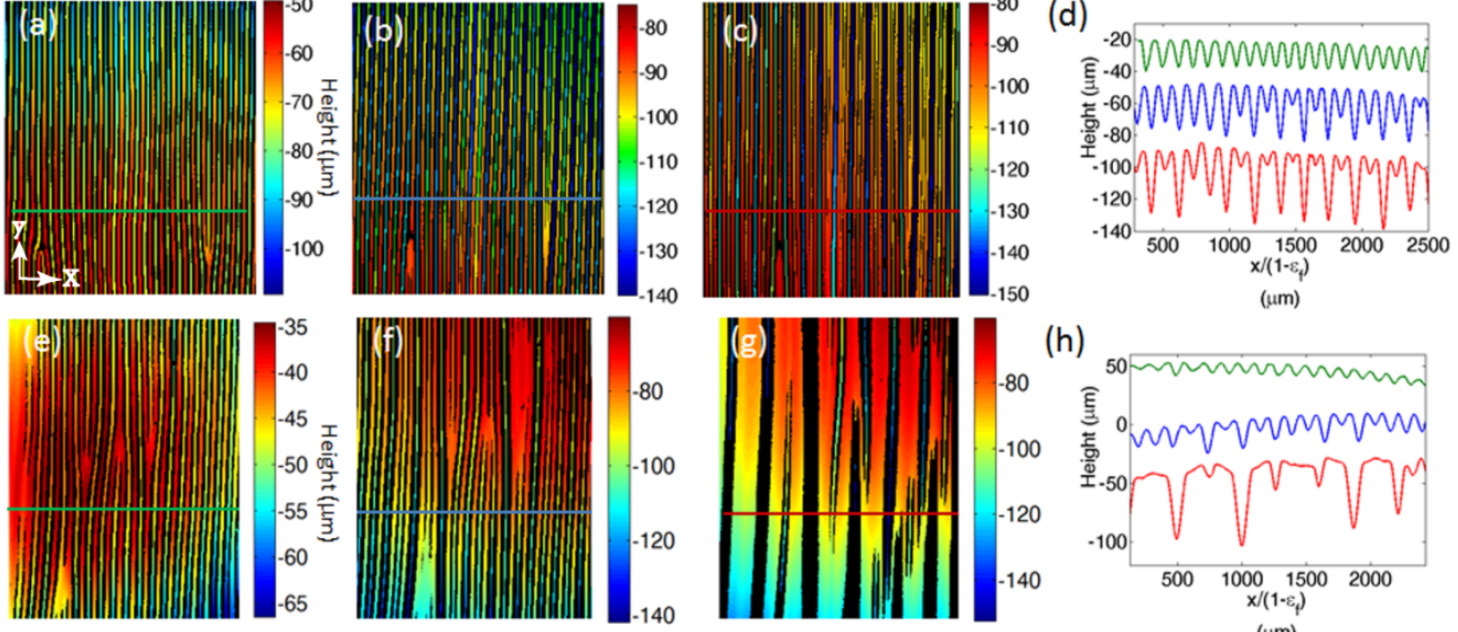

(h)

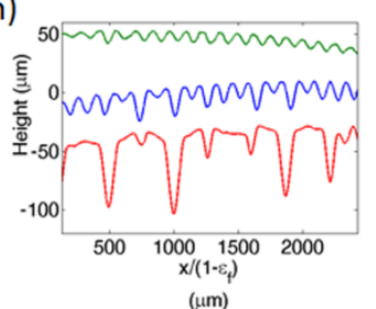

Figure 5. The surface profile of a sample with pre-stretch 1.0 at film strains of (a) 0.083 , (b) 0.16 , (c) 0.23 . (d) One-dimensional profiles at the locations indicated by the solid lines in (a-c). The surface profile of a sample with pre-stretch 0.7 at film strains of (e) 0.045, (f) 0.063 and (g) 0.11 . (h) One-dimensional profiles at the locations indicated by the solid lines in (e-g). 

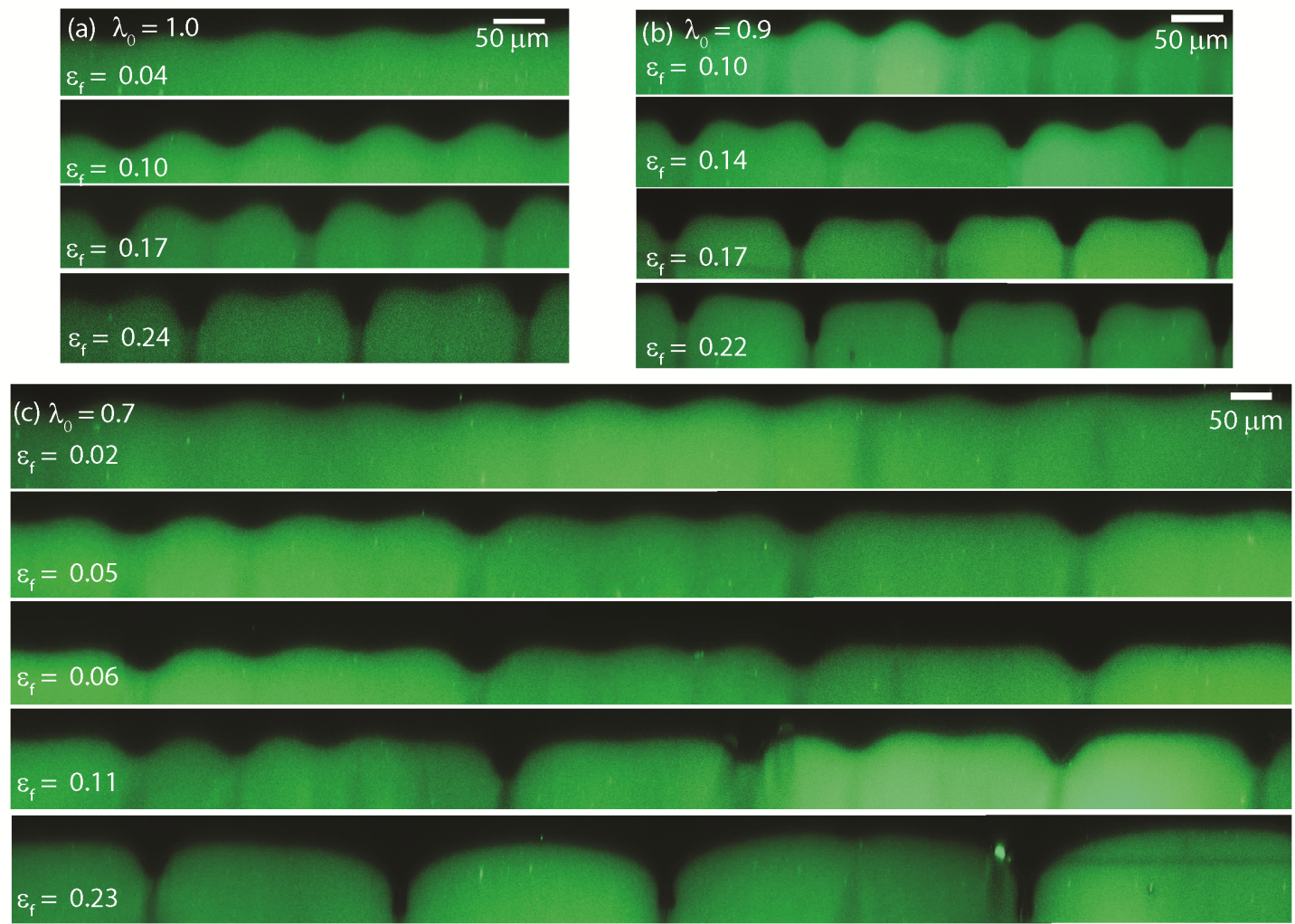

Figure 6. Laser scanning confocal fluorescence cross-sections of compressed bilayers with substrate pre-compression of $\lambda_{0}=$ (a) 1.0 , (b) 0.9 , and (c) 0.7 , showing the emergence of post-wrinkling modes with increasing nominal film compression $\varepsilon_{\mathrm{f}}$. 


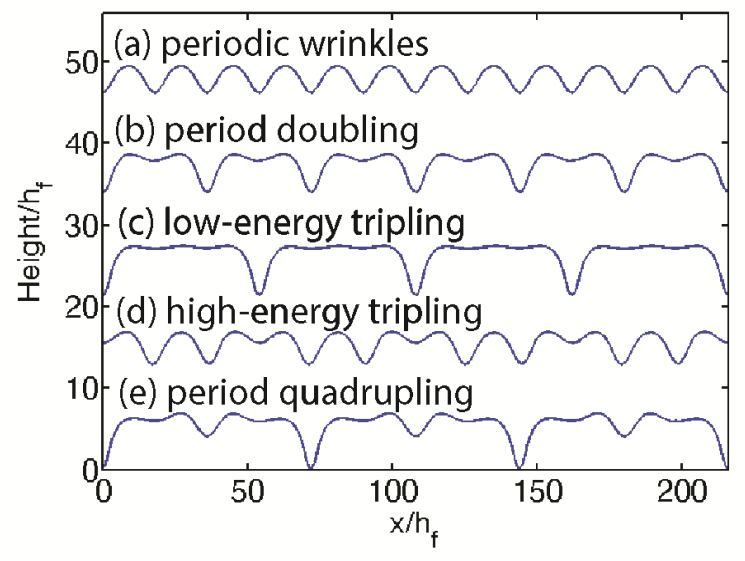

Figure 7. Various post-wrinkling bifurcations obtained by finite element simulation. (a) Wrinkles maintaining the initial periodicity. (b) Period doubling. (c) Low-energy tripling. (d) High-energy tripling. (e) Period quadrupling. The film strain is $\varepsilon_{\mathrm{f}}=0.11$ and the substrate pre-stretch is $\lambda_{0}=0.7$. 


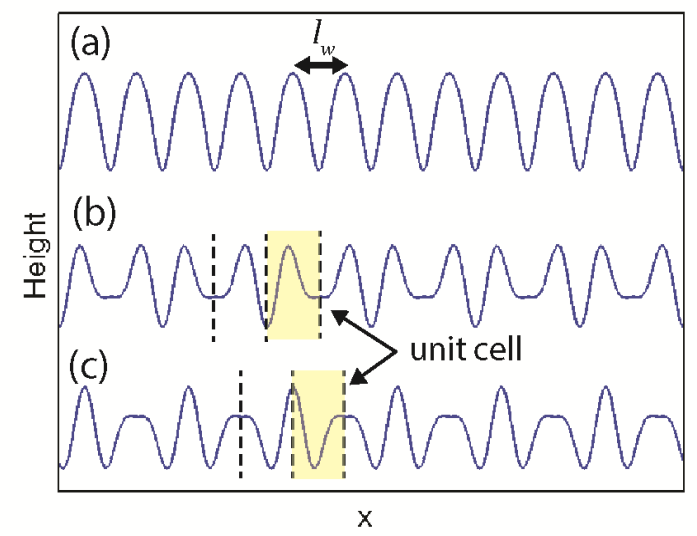

Figure 8. Simulating bifurcation from a periodic wrinkled state to a period-doubled state. (a) The wrinkled state with period $l_{w}$. The finite element simulations use unit cells of width $l_{w}$ of two types. (b) A unit cell with boundaries at the troughs of neighboring wrinkles bifurcates into the period-doubled state at a critical strain $\varepsilon_{\mathrm{pw}}$. (c) The other type of unit cell with boundaries at the peaks of neighboring wrinkles remains in the periodic wrinkled state and does not bifurcate. The simulations start with a nearly flat surface with prescribed imperfections shown in (b) and (c). For visual clarity, the amplitude of the imperfections has been greatly magnified. 

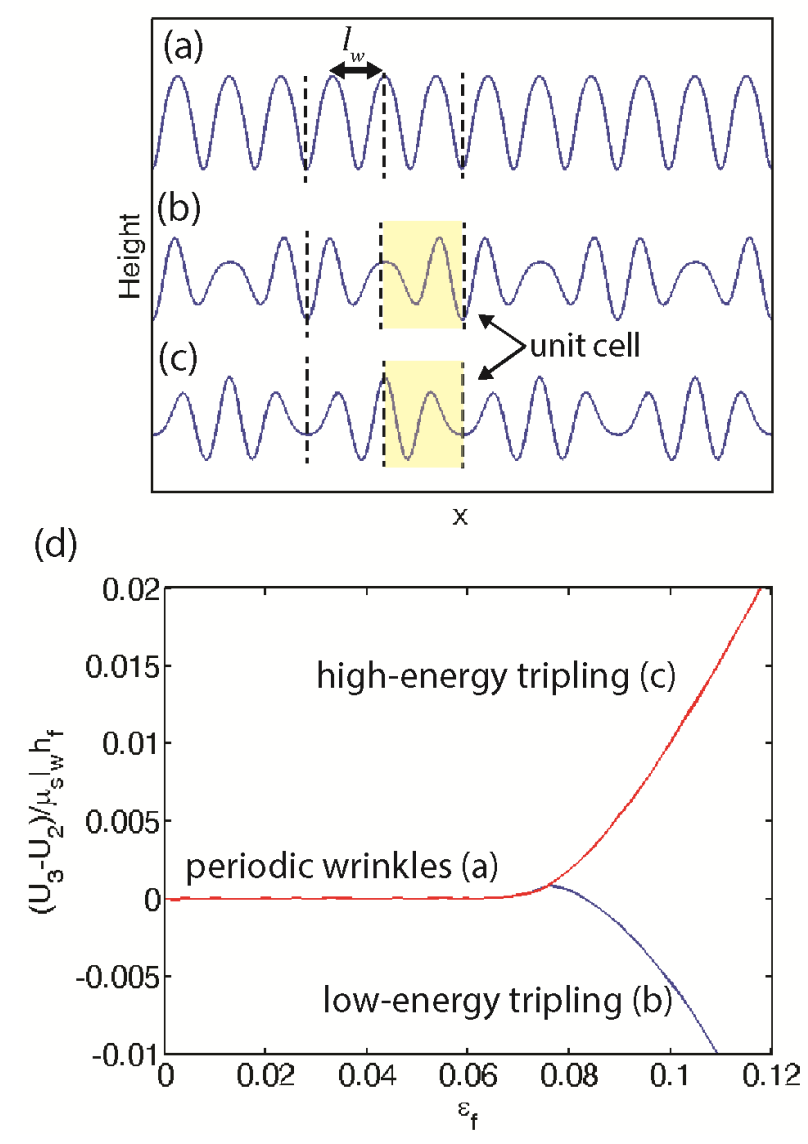

Figure 9. Simulating bifurcation from a periodic wrinkled state to a period-tripled state. (a) The wrinkled state with period $l_{w}$. The finite element simulation uses a unit cell of width $3 l_{w} / 2$, with one boundary at a trough and the other boundary at a peak. This unit cell leads to two modes of period tripling. (b) In one mode, of the three neighboring troughs, one becomes deeper and the other two become shallower. (c) In the other mode, one becomes shallower and the other two become deeper. The simulations start with a nearly flat surface with prescribed imperfections shown in (b) and (c), where the amplitude has been greatly magnified. (d) The difference in the energies of the tripling and doubling modes, $U_{3}-U_{2}$, as a function of the film strain. The pre-stretch is $\lambda_{0}=0.7$. The mode in (b) has a lower energy than the mode in (c). Furthermore, the mode in (b) has a lower energy than the period-doubled state beyond a critical film strain, but the mode in (c) always has a higher energy than the period-doubled state. 


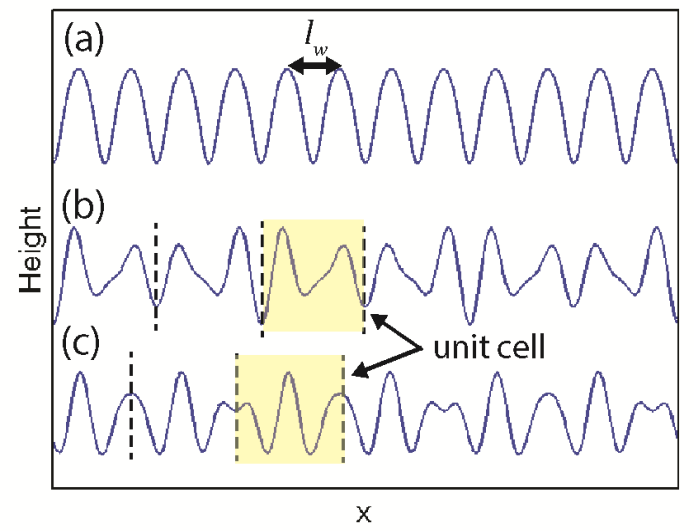

Figure 10. Simulating bifurcation from a periodic wrinkled state to a period-quadrupled state. (a) The wrinkled state with period $l_{w}$. (b) The finite element simulation uses a unit cell of width $2 l_{w}$, with boundaries at two troughs of the wrinkles. With the initial prescribed imperfections shown in (b), magnified in amplitude for clarity, the system undergoes period quadrupling at a critical strain after period doubling. (c) If the boundaries are chosen at the peaks of the wrinkles, period quadrupling cannot happen. 
(a)

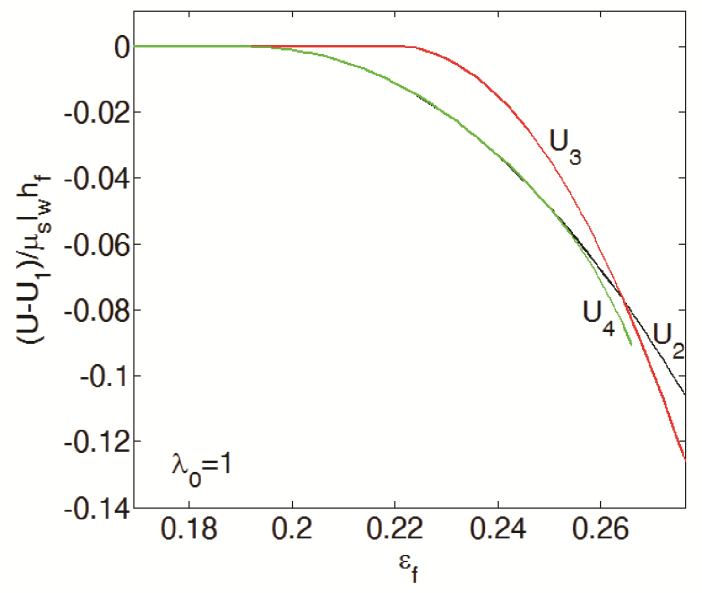

(c)

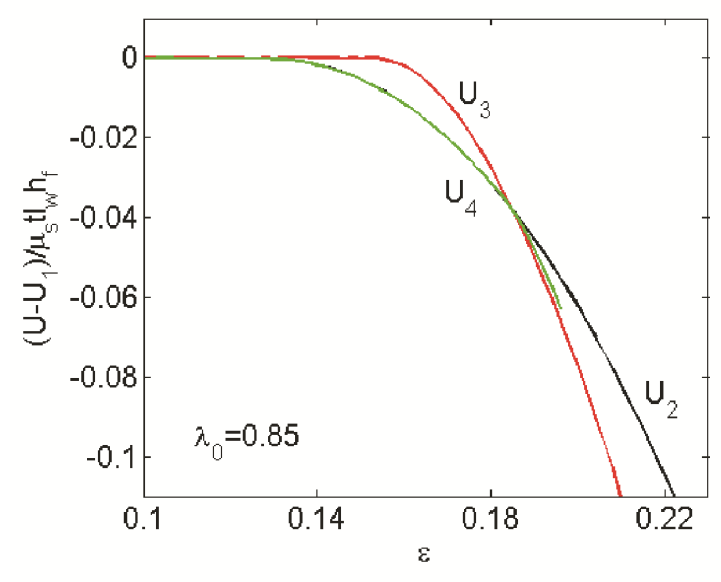

(b)

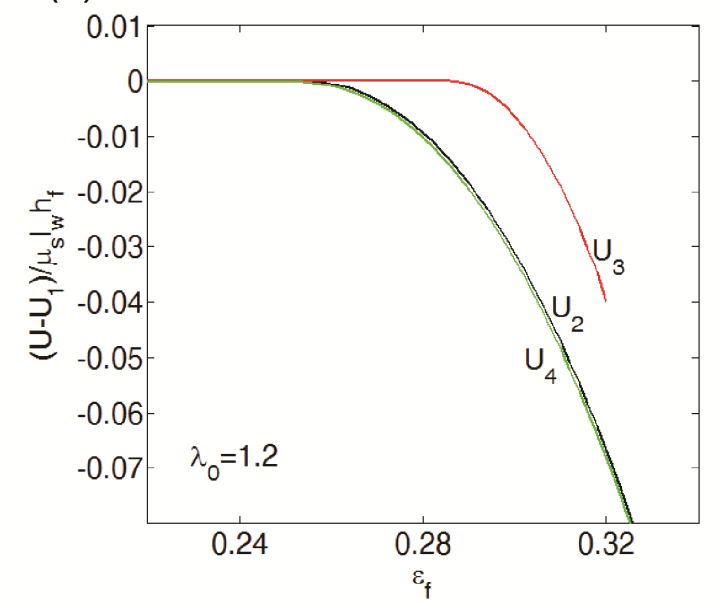

(d)

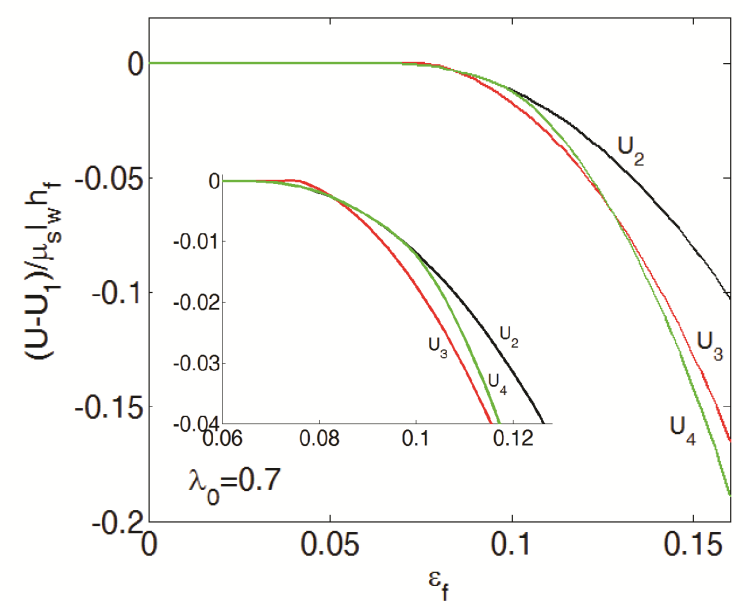

Figure 11. The normalized energy of the period-doubled state $U_{2}$, tripled state $U_{3}$, and quadrupled state $U_{4}$ relative to the energy of the periodic wrinkled state $U_{l}$. The prestretch in the substrate is (a) $\lambda_{0}=1.0$, (b) $\lambda_{0}=1.2$, (c) $\lambda_{0}=0.85$ and (d) $\lambda_{0}=0.7$. The inset in (d) shows a magnification of the region of $\varepsilon_{\mathrm{f}}=0.06-0.13$. 


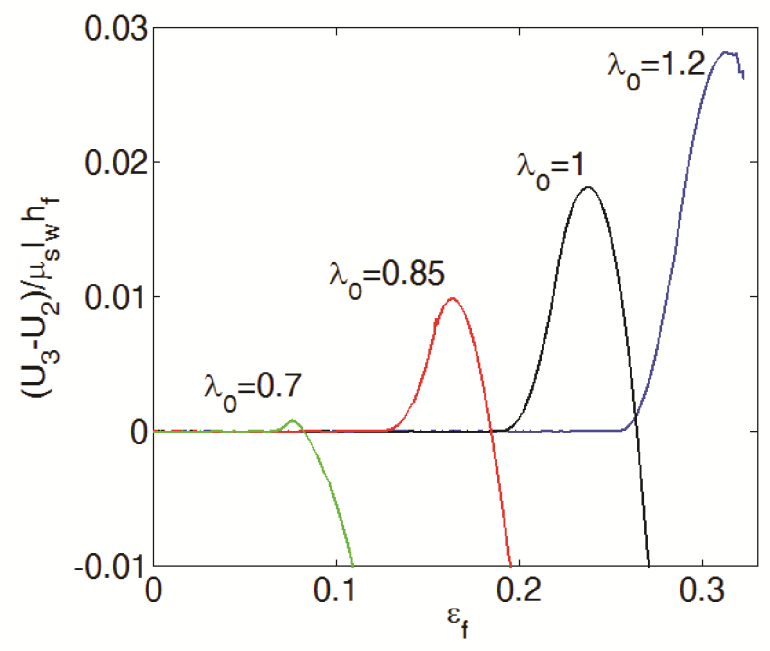

Figure 12. The difference in energy between the period-tripled state $U_{3}$ and the perioddoubled state $U_{2}$ for different values of the substrate pre-stretch $\lambda_{0}$. 


\section{TOC Graphic}

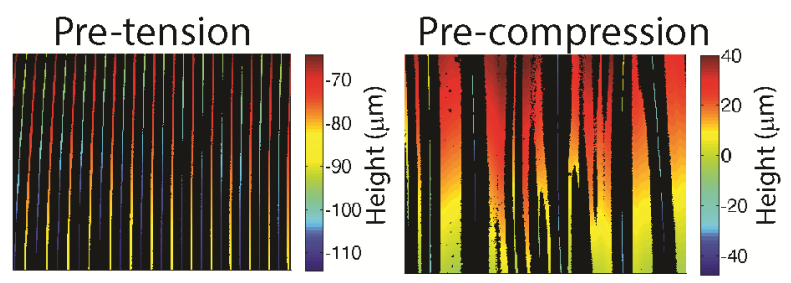

We study the influence of substrate pre-stretch on post-wrinkling bifurcations in bilayers consisting of stiff films on soft substrates by a combination of experiment and modeling. While large pre-tension stabilizes the wrinkled state and therefore delays period doubling, large pre-compression destabilizes wrinkles and gives rise to a chaotic post-wrinkling mode. 